\title{
Influence of N6-benzyladenine and sucrose on in vitro direct regeneration and microrhizome induction of Kaempferia parviflora wall. Ex Baker, an important ethnomedicinal herb of Asia
}

\begin{abstract}
Removal of ciprofloxacin (CIP) pollutant from wastewater using conventional process is particularly challenging due to poor removal efficiency. In this work, CIP was photocatalytically degraded using a porous $\mathrm{ZnO} / \mathrm{SnS} 2$ photocatalyst prepared via microwaves. The influence of process parameters (e.g., $\mathrm{pH}$, catalyst mass and initial CIP concentration) and radical scavengers on visible-light induced degradation of CIP on the catalyst was investigated. From the study, it was found that visible-light induced degradation of CIP on $\mathrm{ZnO} / \mathrm{SnS} 2$ is a surface-mediated process and the reaction kinetics followed the Langmuir-Hinshelwood first-order kinetics. It was found that the optimum condition for CIP degradation was at $\mathrm{pH}$ of 6.1 and catalyst dosage of 500 mg L-1. Higher catalyst dosage however led to a decline in reaction rate due to light scattering effect and reduction in light penetration.
\end{abstract}

Keyword: Visible-light; Ciprofloxacin; Photocatalysis; ZnO/SnS2; Transformation products 